\title{
Ibersid 2009
}

Encuentros Internacionales sobre sistemas de información y documentación

\section{International conference on information and documentation systems}

\section{Addenda}

\section{Número monográfico}

Avances y perspectivas en sistemas

de información y documentación, 2009

\section{Editor:}





\title{
Identidad cultural y gestión de la información y del conocimiento en las organizaciones en cambio
}

\author{
Cultural identity and information and knowledge management in changing organizations
}

\section{Barbara FADEL}

Doutora em História Social pela FFLCH da USP. Professora do Programa de Pós-Graduação em Ciências da Informação da F.F.C. - Unesp, Av. Hygino Muzzi Filho, 737, Marília - São Paulo - Brasil CEP: 17525-900, e do Programa de Pós-Graduação em Desenvolvimento Regional do Centro Universitário de

Franca - Uni-FACEF, Av. Major Nicácio, 2433 - Franca - São Paulo - Brasil - CEP 14.401-135, bafadel@terra.com.br

\section{Resumen}

Esta investigación tiene por objetivo buscar respuestas para la práctica organizacional y los cambios ocurridos en el sistema de gestión a través de la identidad cultural. Tiene como foco los aspectos o puntos comunes de la cultura de las organizaciones, como los valores, las creencias, los mitos, las normas, las innovaciones tecnológicas, entre otros, que se configuran como determinantes del proceso de desarrollo e inserción de la empresa en este nuevo contexto del mundo globalizado. Tiene, aún, el objetivo de detectar, a través de los componentes explícitos e implícitos, la importancia de los valores de la cultura organizacional como camino para la comprensión de la identidad de la organización, apuntando los elementos que deben ser preservados y estableciendo las tendencias y las perspectivas para el sistema de información y gestión. Para alcanzarlos se hace necesario trabajar con abordajes cualitativos y cuantitativos. El universo de investigación será delimitado considerándose la base económica regional del municipio de Franca, tradicionalmente, un fuerte sector de calzados. Hubo la opción, en esta investigación, en trabajar con tres industrias del sector del calzado y sus componentes. La colecta de datos se dará a través de entrevistas abiertas con funcionarios de diferentes niveles de jerarquía. Tendrá por base la escala de valores organizacionales establecida por Tamayo y Gondim (1996), debidamente adaptada a la naturaleza de la investigación. La sistematización y el análisis del conjunto de datos obtenidos permitirán detectar las técnicas y los valores de la cultura organizacional en el desarrollo de la empresa, delineando su identidad y los elementos que deben ser preservados.

Palabras clave: Identidad cultural. Identidad organizacional. Cultura regional. Ambiente de información. Gestión del conocimiento.

\begin{abstract}
The purpose of this research is to seek answers to the organizational practice and the changes that have taken place in the administration system through cultural identity. Its major focus is the common aspects such as values, beliefs, myths, the technological innovations and other aspects that are crucial to the process of development and integration into this new context of a globalized world. It is also to realize, through explicit or implicit components, that the values of the organizational culture will lead to the understanding of the organization identity, pointing out elements to be preserved and setting trends and prospects to the information and administration system. In order to achieve these goals, qualitative and quantitative approaches are necessary. The research will take into account the basis of the regional economy. The city of Franca counts on a strong shoes. Data will be collected through open interviews with employees from several ranks. It will be based on the Tamayo and Gondim's organizational company values scale( (1966) and it will be adapted to the nature of the research. The systematization and analysis of the group of obtained data will make it possible to detect the techniques and values of the organizational culture in the development of the company, delineating its identity and the elements to be preserved.
\end{abstract}

Keywords: Cultural identity. Organizational identity. Regional culture. Information environment. Knowledge administration. 
As organizações enfrentam desafios crescentes, tanto na forma de inserção e interação em sociedades, como no sistema de gestão adotado. Com a globalização, houve quebra de paradigmas e mudanças nas crenças, conceitos e valores da humanidade, novos mercados profissionais surgiram e fizeram com que cada área repensasse suas atividades frente às novas solicitações. Surgem novos enfoques de serviços e produtos. Esse novo sistema provocou, ainda, a multiplicação de diferentes identidades locais como maneira de resistir aos modelos da cultura uniformizante.

Conforme descrevem Fadel e Moraes (2005), o capitalismo passou por um novo período de aprofundamento das descobertas técnicas e científicas, nas duas ultimas décadas do século XX. As inovações, nos campos da informática, telemática, novos materiais e biotecnologia impulsionaram a transformação do padrão de organização da produção e do trabalho nas mais diversas atividades econômicas. Diante das novas possibilidades constituídas e de suas perspectivas, alguns autores têm procurado tratar do conceito do que tem se denominado de terceira revolução tecnológica, na forma de distintos entendimentos, tais como: revolução da informática (HARVEY, 1992; CORIAT, 1988), sociedade informática e/ou da informação (SCHAFF, 1995; LOJKIME, 1995), a sociedade do tempo livre e/ ou a sociedade do conhecimento (MASI, 1999), a sociedade pós-industrial (BELL, 1973; GORZ, 1994) e a economia em rede (CASTELLS, 1998).

Em grande medida, registra-se a presença de uma verdadeira convergência dessa terceira onda de inovação nos meios de comunicação, capaz de alterar profundamente os modos de produção, de trabalho e de vida. As inovações tecnológicas não trilham caminhos se- parados, como a possível convergência entre as três principais tecnologias de comunicação (telefone, televisão e computador), potencializa-se um novo estágio em termos das comunicações, minimizando o poder da geografia, através da redução das distâncias.

A atual revolução tecnológica caracteriza-se não pela centralidade de conhecimentos e informação, mas pela aplicação desses conhecimentos e dessa informação para a geração de novos conhecimentos e de dispositivos de processamento / comunicação da informação, em um ciclo cumulativo de realimentação entre a inovação e seu uso.

Esta sociedade da informação traz novos paradigmas, cria novos caminhos para o desenvolvimento e exige uma nova postura diante das mudanças sociais. Cabe à nova sociedade informacional, emergente desse processo de transformação, planejar ações que conduzam à produção e distribuição de conteúdos que sirvam aos interesses das identidades culturais do país, respeitando sua história, cultura, instituições e relação específica com o capitalismo global e a tecnologia informacional.

Entende-se que identidade cultural é a soma de significados estruturais da vida de um indivíduo ou de um povo, partindo do princípio de que identidade cultural não é única, mas, sim, múltipla. $O$ conceito de Identidade cultural aponta, segundo Teixeira Coelho (1999, p.201), "para um sistema de representação de elementos de simbolização e procedimentos de encenação desses elementos, das relações entre os indivíduos e os grupos e entre estes e seu território de reprodução e produção, seu meio, seu espaço e seu tempo". Ainda, segundo Teixeira Coelho, "este conceito de identidade vem sendo substituído, atualmente, pelo de identificação e 
transforma-se em processo de construção continuada". Para Berger (1996, p.116), "a identidade é socialmente outorgada, socialmente sustentada e socialmente transformada".

As organizações podem ser consideradas um subsistema em interação com um sistema maior e mais abrangente. Para o conhecimento do contexto organizacional faz-se a transposição do conceito de cultura e de identidade para o ambiente organizacional buscando subsídios para o seu conhecimento.

Segundo Caldas e Wood Jr (1997) a idéia de identidade organizacional pode surgir na forma pela qual a organização é percebida, a sua imagem externa, por aqueles com quem ela interage, como, por exemplo, clientes e fornecedores. Pode surgir, também, na forma como seus membros internos, especialmente seus dirigentes, a percebem de forma compartilhada, correspondendo a uma percepção de si mesma ou autopercepção ou auto-imagem. Ou ainda, pode surgir na definição da razão de ser, finalidade, ou propósito da entidade ou do grupo de indivíduos, de modo que a identidade representaria esse propósito existencial.

Para Albert e Wheten, a identidade organizacional compreende as crenças compartilhadas pelos membros sobre o que é central, distintivo e duradouro na organização.

\begin{abstract}
O critério de centralidade aponta as características vistas como essência da organização. O critério da distintividade aponta os elementos que distinguiria uma organização das outras com as quais poderia ser comparada. O critério de continuidade temporal ressalta as características estáveis no tempo. (1985, p.265)
\end{abstract}

Esses três critérios seriam, segundo os autores, cada um necessário e, como conjunto, suficientes para definir identidade organizacional como conceito científico.
De tal forma que a identidade é tudo que torna algo único. No caso de uma organização, podemos entender sua identidade institucional como o conjunto de suas características próprias e exclusivas, refletindo sua personalidade. Compõe-se das definições do seu negócio, da sua missão, visão e valores;

- Negócio é o âmbito de atuação da empresa. Representa o espaço que a organização deseja ocupar em relação às demandas da sociedade. Permite orientar as ações de todos com os propósitos da alta administração.

- Missão de uma organização descreve a natureza e o conceito de suas atividades, ressaltando a filosofia que deve orientar sua relação com os usuários/clientes e organizações relacionadas.

- A visão de futuro de uma organização expressa o entendimento sobre o seu papel no futuro, tendo em vista sua missão e seus possíveis espaços de intervenção. Ela tem o objetivo de clarificar a direção da mudança organizacional, permitindo a tomada de iniciativas que possam, aos poucos, ir colaborando na construção da nova organização.

- Os valores são definidos como um conjunto de convencimentos acerca do que é essencial para a instituição: os princípios que são importantes e que definem aquilo que os seus funcionários devem prestar atenção. Princípios ou crenças e valores constituem a base de sustentação de todas as ações de administradores e empregados da instituição. Representam o que se acredita como certo, correto e leal.

A cultura organizacional tem sido utilizada nos estudos do ambiente organizacional. Por tratar do comportamento humano nas organizações, tem servido 
como base para explicar as ações coletivas ou, mesmo, individuais que lá ocorrem. A cultura organizacional fomenta, também, a gestão do conhecimento na organização, pois envolve, principalmente, o trabalho com o relacionamento humano e seus vários elementos intangíveis. Visualizar a cultura como uma forma de representação da realidade enfatiza a maneira pela qual as pessoas entendem a organização. Por outro lado, a identificação dos aspectos da cultura organizacional na própria rotina da organização, no significado dado para cada ato realizado, no fundamento do agir, assim como nas atitudes, seria calcada na interpretação que a pessoa faz do ambiente organizacional.

Tendo por base as diferentes interpretações, pode-se mapear os elementos da cultura organizacional, como as crenças, histórias, mitos, heróis, tabus, normas e rituais. Esses elementos, presentes no ambiente organizacional, podem ser assimilados pela integração ao grupo, num processo de socialização, como pode ser, contínua e individualmente, construído e incorporado ao ambiente organizacional. Também são resultados da forma de interpretação da realidade, a estrutura organizacional, as regras, as políticas, os valores, os objetivos, as missões e as descrições de cargos, todos eles influenciados pela forma como o coletivo humano interpreta a organização. (Morgan, 1996)

Schein (1992) interpreta a cultura como sendo formada pelo conjunto de pressupostos básicos que um grupo inventou, descobriu ou desenvolveu, ao aprender a lidar com os problemas de adaptação externa e integração interna, e que funcionaram bem o suficiente, sendo, por isso, perpetuados pelo grupo.

Sendo a cultura organizacional o resultado de um longo processo de existência de uma organização, a necessidade de mudança pode se tornar um processo demorado e doloroso. São os valores da organização que definem a sua cultura organizacional; assim, esses valores devem ser compatíveis com a adaptação e a orientação de mudança, expansão e crescimento, aliados à perseguição de uma perfeita técnica. Nos processos de mudança dos sistemas de gestão, questiona-se o que mudar, o que preservar e como mudar.

Considerando a cultura organizacional como uma interpretação coletiva da realidade, ela deve oferecer os caminhos para se trabalhar a gestão do conhecimento na organização, que, nesta ultima década, tem sido tão discutido pela maioria dos gerentes e executivos empresariais. A sua estrutura, incorporada aos recursos humanos da organização, ofereceria as condições necessárias à geração, ao uso e ao compartilhamento do conhecimento, possibilitando a sua gestão. Os elementos que possibilitariam a gestão do conhecimento podem ser detectados através das crenças, pressupostos, posturas, atividades que se caracterizam por uma postura proativa, aberta, abrangente e atuante.

O domínio psíquico seria o aspecto central da postura ou interpretação que os membros da organização têm com relação ao conhecimento. Assim, o conhecimento tácito, segundo Nonaka e Takeuchi (1997), encontra-se diretamente relacionado com a cultura organizacional por ser composto, em grande parte, pelos elementos presentes na psique do indivíduo, e fazem parte de sua noção de como interpretar a realidade, fator necessário à construção da ação. $O$ agir também necessita da participação do conhecimento explicito, o que o faz necessário para a construção do conhecimento pleno, tácito mais explícito, que envolve os aspectos essenciais da cultura organizacional presentes na psique dos membros da or- 
ganização. Trabalhar os elementos psíquicos presentes na organização auxilia na forma de trabalhar o cerne da gestão do conhecimento. O domínio psíquico seria o aspecto central da postura ou interpretação que os membros da organização têm com relação ao conhecimento.

Ao buscar respostas para a prática organizacional e as mudanças que virão a ocorrer no sistema de gestão da empresa, é necessário enfatizar a importância e evidenciar os valores da cultura organizacional que darão suporte e apoio às mesmas. As inovações tecnológicas e organizacionais normalmente apresentam traços comuns e identificam um desenvolvimento da cultura organizacional que reflete uma identidade $e$ permite discutir a cultura de grupos. A socialização é discutida a partir do cotidiano e da análise do comportamento das pessoas, criando um espaço para que elas possam aprender e crescer continuamente. $\mathrm{Na}$ era atual, do conhecimento e da globalização, há necessidade de se estabelecer as tendências e perspectivas que servirão de dados, informações e conhecimento para a participação da organização em toda essa evolução, com estratégia, trilhando a cultura da aprendizagem, que levará às mudanças na dinâmica da organização, dentro do contexto do conhecimento e da memória organizacional.

Esta pesquisa tem por objetivo buscar respostas para a prática organizacional e as mudanças ocorridas no sistema de gestão, através da identidade cultural. Tem como foco os aspectos ou pontos comuns da cultura das organizações, como os valores, as crenças, os mitos, as normas, as inovações tecnológicas, entre outros, que se configuram como determinantes do processo de desenvolvimento e inserção da empresa neste novo contexto de mundo globalizado.
A organização adquire uma identidade somente ao tornar-se infundida de valor. Como infundir valor a uma organização nessa sociedade globalizada? Como construir uma identidade cultural em organizações transnacionais ou organizações que participaram de aquisições e fusões empresariais? Detectar, através dos componentes explícitos e dos implícitos, a importância e os valores da cultura organizacional é o caminho para a compreensão da identidade da organização, apontando os elementos a serem preservados e estabelecendo as tendências e perspectivas para o sistema de informação e gestão.

Serão apresentados os diferentes tipos de empresas existentes na sociedade atual, das quais destacamos a empresa doméstica ou nacional, empresa internacional, empresa multinacional, empresa transnacional e empresa global.

O universo da pesquisa será delimitado levando-se em conta a base econômica regional. O município de Franca conta, tradicionalmente, com forte setor calçadista e seus componentes, cultura do café, extração de diamante, bacia leiteira, e, mais recentemente, com indústrias de confecção e de cosméticos.

Optou-se, nesta pesquisa, por trabalhar com três indústrias do setor calçadista e seus componentes. Para a seleção dessas indústrias, serão estabelecidos critérios, levando-se em conta sua história, seu desenvolvimento e sua participação no mercado interno e externo. A coleta de dados dar-se-á através de entrevistas abertas, com funcionários de diferentes níveis hierárquicos. Terá por base a escala de valores organizacionais estabelecida por Tamayo e Gondim (1996), devidamente adaptada à natureza da pesquisa e da empresa, para a verificação dos que forem mais significativos. Utilizar-se-á, ainda, como fonte primária de dados, textos, normas, regulamentos e produtos de divulgação 
da empresa. Os valores organizacionais serão agrupados pelos fatores e o resultado obtido deverá representar a força de cada valor e de seu correspondente fator, segundo a percepção dos sujeitos envolvidos. A sistematização e a análise do conjunto de dados obtidos pelas abordagens qualitativas e quantitativas permitirão detectar as técnicas e os valores da cultura organizacional no desenvolvimento da empresa, delineando a sua identidade e os elementos a serem preservados.

\section{Referencias}

Arantes, A. N. Sistemas de gestão empresarial. São Paulo: Atlas, 1994. 440p.

Bowditch, J. L. Buono, A. F. Elementos de comportamento organizacional. São Paulo: Pioneira, 1992. 305 p.

Coelho, Teixeira. Dicionário crítico de política cultural. $2^{\mathrm{a}}$ ed. São Paulo: FAPESP; Ed. lluminuras, 1999.
Collins, J. C.; Forras, J. I. Feitas para durar. Rio de Janeiro: Rocco, 1995. 209p.

Crainer, S. Os revolucionários da administração. São Paulo: Negócio Editora, 1999. 333p,

Fadel, B.; Moraes, C. R. B. As ondas de inovação tecnológicas. // FACEF Pesquisa, v.8, n.1, p.34-40, 2005.

Fleury, M. T. L.; Ficher, R. M. Cultura e poder nas organizações. 2. ed. São Paulo: Atlas, 1996. 170p.

Freitas, M. E. Cultura organizacional - formação, tipologias e impactos. 2. ed. São Paulo: Makron, 1991. 178p.

Hall, S. A identidade cultural na pós-modernidade. Rio de Janeiro: DP\&A, 2001.

Machado, H. V. A identidade e o contexto organizacional: perspectivas de análise. RAC. Edição especial, p. 51-73, 2003.

Nonaka, I., Takeuchi H. Criação de conhecimento na empresa. 7. ed. Rio de Janeiro: Campus, 1997. 358p

Schein, E. H. Organizational culture and leadership. San Francisco: Jossey-Bass Publisher, 1992.

Tamayo, A.; Gondim, M.G.C. Escala de valores organizacionais. Revista de Administração da USP, v.31, n.2, p.62-72, 1996

Teixeira, F. J. Gerenciando conhecimento. São Paulo: Senac, 2000.

$192 p$ 